\title{
Research Article \\ Effects of Electroacupuncture Treatment on Bone Cancer Pain Model with Morphine Tolerance
}

\author{
Lei Sima, Bifa Fan, Longtao Yan, and Yuan Shui \\ National Pain Management and Research Center, China-Japan Friendship Hospital, Beijing, China \\ Correspondence should be addressed to Lei Sima; simalei75@126.com
}

Received 3 June 2016; Revised 9 August 2016; Accepted 22 August 2016

Academic Editor: Ke Ren

Copyright (C) 2016 Lei Sima et al. This is an open access article distributed under the Creative Commons Attribution License, which permits unrestricted use, distribution, and reproduction in any medium, provided the original work is properly cited.

\begin{abstract}
Objective. To explore the efficacy of electroacupuncture treatment in cancer induced bone pain (CIBP) rat model with morphine tolerance and explore changes of calcitonin-gene related peptide (CGRP) expression in dorsal root ganglion (DRG). Methods. Forty SD rats were divided into five groups: sham, CIBP (B), CIBP + morphine (BM), CIBP + electroacupuncture (BE), and CIBP + morphine + electroacupuncture (BME). B, BM, BE, and BME groups were prepared CIBP model. The latter three groups then accepted morphine, electroacupuncture, and morphine combined electroacupuncture, separately, nine days consecutively (M1 to M9). Mechanical withdraw threshold (MWT) was evaluated. Results. BE group only had differences in M1, M2, and M3 compared to B group $(P<0.01)$. From M5, BM group showed significantly decreased MWT. Electroacupuncture could obtain analgesic effects only at early stage (M1 to M5). From M5 to M9, BME had the differences with BM group $(P<0.01)$. IOD value of CGRP in BM and $\mathrm{BME}$ was substantially less than in B group. CGRP in BME was significantly lower than that in BM group $(P<0.01)$. Conclusion. When used in combination with electroacupuncture, morphine could result in improving analgesic effects and reducing tolerance. CGRP may be associated with pain behaviors.
\end{abstract}

\section{Introduction}

The incidence of bone metastases from commonly found malignant tumors including lung, breast, and prostate cancers is as high as $30-40 \%, 65-75 \%$, and $65-75 \%$ [1], respectively. As there are rich sensory nerves on the periosteum, the invasion of the tumor to the periosteum will cause severe nociceptive pain, while the damaged sensory nerves could be further sensitized and remodeled, which could result in neuropathic pain [2]. Therefore, cancer induced bone pain (CIBP) includes both nociceptive pain and neuropathic pain. So the mechanism has been considered very complex. Currently, the cancer pain models used for the investigations mainly focused on the bone metastases. Opioids that are represented by morphine are the most used and most effective drug for the treatment of cancer pain; however, the use of morphine is greatly constricted by the high incidence of morphine tolerance. In addition, clinical practices show that patients with bone cancer pain are easier to develop morphine tolerance, which has been considered as one of the major causes of uncontrollable pain in the patients with advanced cancers [3].

$\mu$-Opioid receptor (MOR) could not be endocytosed to allow desensitization and resensitization, which is associated with the downregulation of the MOR numbers on the cellular membrane [4]. The endocytosis of the MOR is mediated by the neurotransmitters including calcitoningene related peptide (CGRP) and substance P (SP), which play important roles in the negative regulation of the opioid tolerance [5]. Treating cancer pain with acupuncture has been recommended by the NCCN cancer treatment guideline, the most used guideline for the treatment of tumors all over the world, issued by the National Cancer Institute (NCI) of USA. In recent years, treating cancer pain $[6,7]$, especially the bone cancer pain, with acupuncture has provided a lot of valuable evidence $[8,9]$. However, no study focused on the effects of electroacupuncture treatment on bone cancer painmorphine tolerance has been published to date. 


\section{Materials and Methods}

2.1. Animals and Equipment. Prior permission for animal experimentation was obtained from the Institutional Animal Ethics Committee. Forty SPF grade female SD rats with the body weights of $180-201 \mathrm{~g}$ were obtained from the Vital River Experimental Animal Center (Beijing, China, number SCXK9 (Jing) 2007-0001). Walker 256 breast cancer cells were obtained from the cancer institute of the Peking Union Medical College. Morphine was purchased from the first pharmaceutical company of Shenyang. Polyclonal rabbit anti-CGRP antibody (1:5000) was obtained from DAKO. Von Frey fibre was from Noah Coast Medical Company (USA). Han's electroacupuncture apparatus (LH202) with the parameters of $2 / 100 \mathrm{~Hz}$ and $1 \mathrm{~mA}$ was used.

2.2. Inducing the Bone Cancer Pain-Morphine Tolerance Rat Models. The rat model of bone cancer pain-morphine tolerance was induced according to the methods described in previous studies. In brief, intraperitoneal injection of Walker 256 breast cancer cells was performed for the rats, and then the ascites was collected on day 7 after the injection. The cell density was adjusted to $4 \times 10^{2} / \mu \mathrm{L}$; thermal-inactivated cancer cells were also prepared. Intrathecal catheterization was performed for the rats, and lidocaine was used to evaluate whether the catheterization was successful.

The rats successfully catheterized were divided into 5 groups on day 5 after the catheterization using the random number table in each as follows: sham group, bone cancer pain group (B group), bone cancer pain plus morphine tolerance group (BM group), bone cancer pain plus electroacupuncture group (BE group), and bone cancer pain plus morphine tolerance plus electroacupuncture group (BME group). Tibia cancer pain model was induced for the rats in the $\mathrm{B}, \mathrm{BM}, \mathrm{BE}$, and $\mathrm{BME}$ groups by injecting $10 \mu \mathrm{L}$ of human Walker 256 breast cancer cells into the bone marrow cavity at the upper part of the right tibia.

Our previous studies have demonstrated that bone cancer pain would occur on day 6 after the induction; thus, since day 7 after the induction, intrathecal injection of morphine $(20 \mu \mathrm{g} / \mathrm{kg}, 2$ times/d, for 9 continuous days) for the rats in the $\mathrm{BM}$ group, electroacupuncture treatment for the rats in the $\mathrm{BE}$ group, and morphine plus electroacupuncture treatment for the rats in the BME group were performed, respectively, while, for the rats in the sham group, the upper part of the right tibia was exposed and intrathecal injection of the same volume of normal saline was performed since day 7.

2.3. Treatments. Zusanli (ST36) and Sanyinjiao (SP6) acupoints that were commonly used for the treatment of cancer pain were selected in this study [10]. For the rats in the BME and BE groups, $2 / 100 \mathrm{~Hz}$ high/low frequency alternating current Han's electroacupuncture apparatus was used for the treatment, with the stimulation intensities of $0.5,1$, and $1.5 \mathrm{~mA}$ that were increased every $10 \mathrm{~min}$. The stimulation time was $30 \mathrm{~min}$ for each stimulation intensity. The treatment was started on day 7 after the induction with the frequency of 1 time/day for 9 continuous days.
2.4. Measuring the Pain Behaviors. The mechanical withdrawal threshold (MWT) of the rats was measured before the injection of the cancer cells (baseline, T0), at 3 and 6 days after the injection (T3 and T6), and at 1, 3, 5, 7, and 9 days after the morphine/electroacupuncture treatments (M1, M3, M5, M7, and M9). For von Frey testing, the rats were placed in enclosures on an elevated wire mesh floor, and mechanical allodynia was assessed using a dynamic plantar aesthesiometer (Ugo Basile, Stoelting, IL, USA). A metal probe $(0.5 \mathrm{~mm}$ diameter $)$ was directed against the hind paw pad, and an upward force was exerted. The force required to elicit a withdrawal response was measured in grams and automatically registered when the paw was withdrawn or the preset cut-off was reached $(15 \mathrm{~g})$.

2.5. Measuring CGRP Expression in the Dorsal Root Ganglion by Immunohistochemical Examinations. After the last measurement of the pain behaviors, the rats were anaesthetized with pentobarbital injection $(100 \mathrm{mg} / \mathrm{kg}$ ) and transcardially perfused with $4 \%$ paraformaldehyde in $0.1 \mathrm{M}$ phosphate buffer saline (PBS). The dorsal root ganglion (DRG) was dissected out and preserved in PBS for a further period of 3 days. Cryostat sections ( $20 \mu$ thick) were cut in the transverse plane at $-20^{\circ} \mathrm{C}$ and collected as free-floating sections in $0.1 \mathrm{M}$ phosphate buffer saline (PBS). An average of 11-12 sections per rat was collected. Later, the sections were processed for immunohistochemical localization of CGRP. Following this, sections were directly exposed to primary antibody against CGRP (1: 400; Calbiochem, USA) for $48 \mathrm{~h}$. CGRP expression was visualized by the ABC method (Vector Laboratories, USA) using 3,3-diaminobenzidine (DAB) tetrachloride $(\mathrm{DAB})$ as the chromogen. Finally, the sections were taken on slides and dehydrated followed by clearing.

Images of the stained sections were captured under the microscope and the images were saved in ProgRes Capture Pro 2.7 Image analysis software (Jenoptik, Germany). The area of the CGRP expression in these sections, which corresponded to the superficial part of the DRG, was selected by Image Pro Plus software to calculate the integral optical density (IOD).

2.6. Statistical Analysis. SPSS 11.0 software was used for the statistical analysis. All the data were described as means \pm standard divisions. One-way analysis of variance (ANOVA) and $t$-test were used for the comparisons. $P<0.05$ was considered statistically significant.

\section{Results}

3.1. MWT of the Rats. On day 6 (T6) after the injection of the cancer cells, the MWT in all the four groups of the rats decreased significantly due to the CIBP caused by bone cancer, and the differences with the sham group were statistically significant $(P<0.01)$. From M1 to M9, all the groups with treatment had the differences compared with $\mathrm{B}$ group $(P<0.01)$. BE group only had differences in M1, M2, and M3 compared to B group $(P<0.01)$. From M5, BM group showed significant decreased MWT due to morphine tolerance. $\mathrm{ED}_{50}$ value was $70.4 \pm 9.5 \mu \mathrm{g}$ for cumulative 
TABLE 1: Mechanical withdrawal threshold (MWT) of the rats during bone cancer pain and treatment.

\begin{tabular}{|c|c|c|c|c|c|c|c|c|c|}
\hline & T0 & $\mathrm{T} 1$ & T3 & T6 & M1 & M3 & M5 & M7 & M9 \\
\hline Sham & $12.3 \pm 2.2$ & $12.4 \pm 2.7$ & $12.8 \pm 2.2$ & $12.5 \pm 2.5$ & $12.3 \pm 2.6$ & $12.5 \pm 2.5$ & $12.3 \pm 2.7$ & $12.2 \pm 2.6$ & $12.5 \pm 2.2$ \\
\hline BM group & $12.7 \pm 2.8$ & $12.7 \pm 2.5$ & $12.4 \pm 2.1$ & $8.5 \pm 1.0^{\mathrm{a}}$ & $10.7 \pm 0.7^{\mathrm{ab}}$ & $11.4 \pm 2.0^{\mathrm{ab}}$ & $8.7 \pm 0.6^{\mathrm{ab}}$ & $8.5 \pm 2.1^{\mathrm{ab}}$ & $8.3 \pm 2.5^{\mathrm{ab}}$ \\
\hline BME group & $12.4 \pm 2.4$ & $12.5 \pm 2.2$ & $12.5 \pm 2.5$ & $8.4 \pm 1.8^{\mathrm{a}}$ & $10.9 \pm 0.9^{\mathrm{ab}}$ & $11.1 \pm 2.1^{\mathrm{ab}}$ & $10.9 \pm 0.8^{\mathrm{abc}}$ & $10.2 \pm 1.8^{\mathrm{abc}}$ & $9.7 \pm 2.7^{\mathrm{abc}}$ \\
\hline $\mathrm{BE}$ group & $12.6 \pm 2.5$ & $12.6 \pm 2.5$ & $12.3 \pm 2.7$ & $8.6 \pm 1.8^{\mathrm{a}}$ & $6.2 \pm 0.9^{\mathrm{ab}}$ & $6.0 \pm 1.7^{\mathrm{ab}}$ & $6.2 \pm 1.5^{\mathrm{ab}}$ & $5.9 \pm 1.4^{\mathrm{a}}$ & $6.1 \pm 0.8^{\mathrm{a}}$ \\
\hline B group & $12.5 \pm 2.2$ & $12.5 \pm 2.4$ & $12.4 \pm 2.4$ & $8.7 \pm 2.0^{\mathrm{a}}$ & $5.3 \pm 1.5^{\mathrm{a}}$ & $5.1 \pm 1.6^{\mathrm{a}}$ & $5.1 \pm 1.5^{\mathrm{a}}$ & $5.6 \pm 1.4^{\mathrm{a}}$ & $5.7 \pm 1.7^{\mathrm{a}}$ \\
\hline
\end{tabular}

${ }^{\mathrm{a}}$ Compared with sham, $P<0.01$; ${ }^{\mathrm{b}}$ compared with B group, $P<0.01$; ${ }^{\mathrm{c}}$ compared with BM group, $P<0.01$.

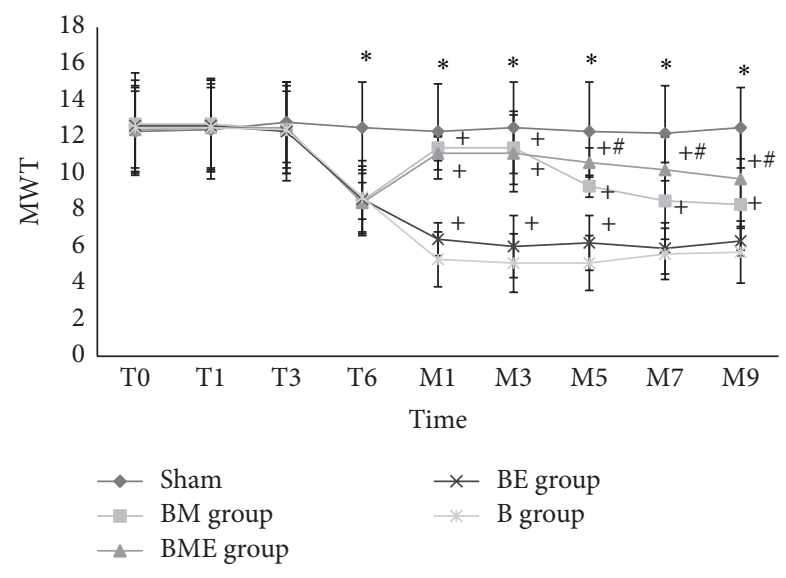

FIGURE 1: MWT of the rats during bone cancer pain and treatment. ${ }^{*}$ Significant difference from sham group since day $6(P<0.01)$. ${ }^{+}$Significant difference from B group since morphine/EA treatment $(P<0.01)$. " Significant difference from BM group since morphine tolerance $(P<0.01)$.

dose after morphine tolerance. Electroacupuncture treatment alone for bone cancer pain could obtain analgesic effects only at early stage (M1 to M5). But the analgesic effect was much less than morphine. The longer electroacupuncture treatment (M7 to M9) showed analgesic tolerance too. From M5 to M9, BME group had differences compared with the BM group $(P<0.01)$; see Table 1 and Figure 1.

3.2. CGRP Expression and the IOD Value. The immunoreactive substances of CGRP peptide are mainly at the margins of the DRG, while the staining of the CGRP in B group and $\mathrm{BE}$ group was evidently deeper than $\mathrm{B}$ group. The IOD value of CGRP in BM and BME group was substantially less than in $B$ group. The IOD value of the CGRP in the BME group was significantly lower than that in BM group $(P<0.01)$, as shown in Figures 2 and 3.

\section{Discussion}

According to the theories of zangfu and meridians, masters of traditional Chinese medicine proposed the theory of "Qi and blood running" to explain pain, which suggests "stagnation leading to pain." The principle of acupuncture analgesia is soothing the channels and improving collateral circulation. Western medicine suggests that "acupuncture could result in analgesic effects via stimulating the release of endogenous

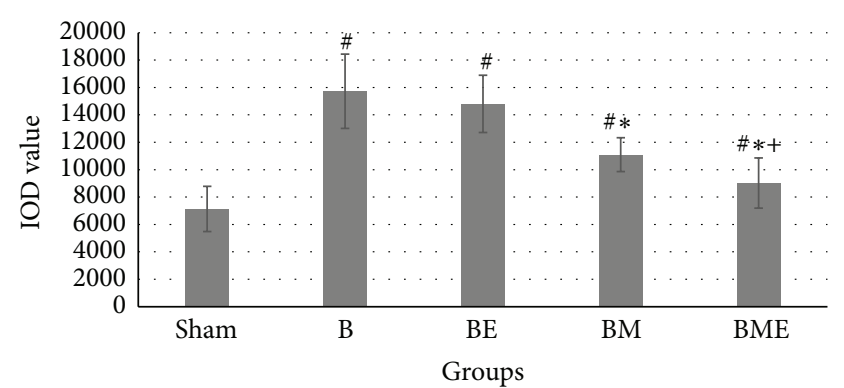

FIGURE 2: IOD value of CGRP in dorsal root ganglion of the rats. ${ }^{\#}$ Compared with sham, $P<0.01$. ${ }^{*}$ Compared with B group, $P<$ $0.01{ }^{+}$Compared with BM group, $P<0.01$.

opioid peptides," and further suggests that stimulation at low frequency $(2 \mathrm{~Hz})$ could accelerate the synthesis and release of encephalin and endorphin, while stimulation at high frequency $(100 \mathrm{~Hz})$ could promote the synthesis and release of dynorphin. Stimulation with high-frequency electroacupuncture $(2 / 100 \mathrm{~Hz})$ could accelerate the synthesis and release of all these three opioid peptides [11-13]. The encephalin, endorphin, and dynorphin are the endogenous ligand for $\mu$-, $\delta$-, and $\kappa$-opioid receptor, respectively [14].

The findings of the present study showed that the pain behavior changes since day 6 after the injection of cancer cells, which was in agreement with the time reported in previous studies. Our findings showed that, after injection of morphine for 5 consecutive days, the MWT of the rats decreased, which suggested the occurrence of bone cancer pain-morphine tolerance. We speculated that the mechanisms involved could be associated with the overrelease of the SP and CGRP in the DRG. With more pain causing neurotransmitters release from the presynaptic membrane into the synaptic cleft, the analgesic effects of morphine reduced substantially.

In addition to this, the present study further demonstrated that electroacupuncture treatment is effective in treating morphine tolerance. The analgesic effects of using electroacupuncture treatment alone in the early stage of analgesia (M1-M3) were lower than using morphine alone, while the effects of using morphine plus electroacupuncture treatment were similar to using morphine alone, suggesting that the analgesic effects from morphine were dominant in the early stage of analgesia. However, morphine tolerance occurred in the late stage of analgesia (M5-M9), while the tolerance in the BME group was lower than in the BM group, suggesting that electroacupuncture treatment could effectively reduce the morphine tolerance and increase the MWT. 

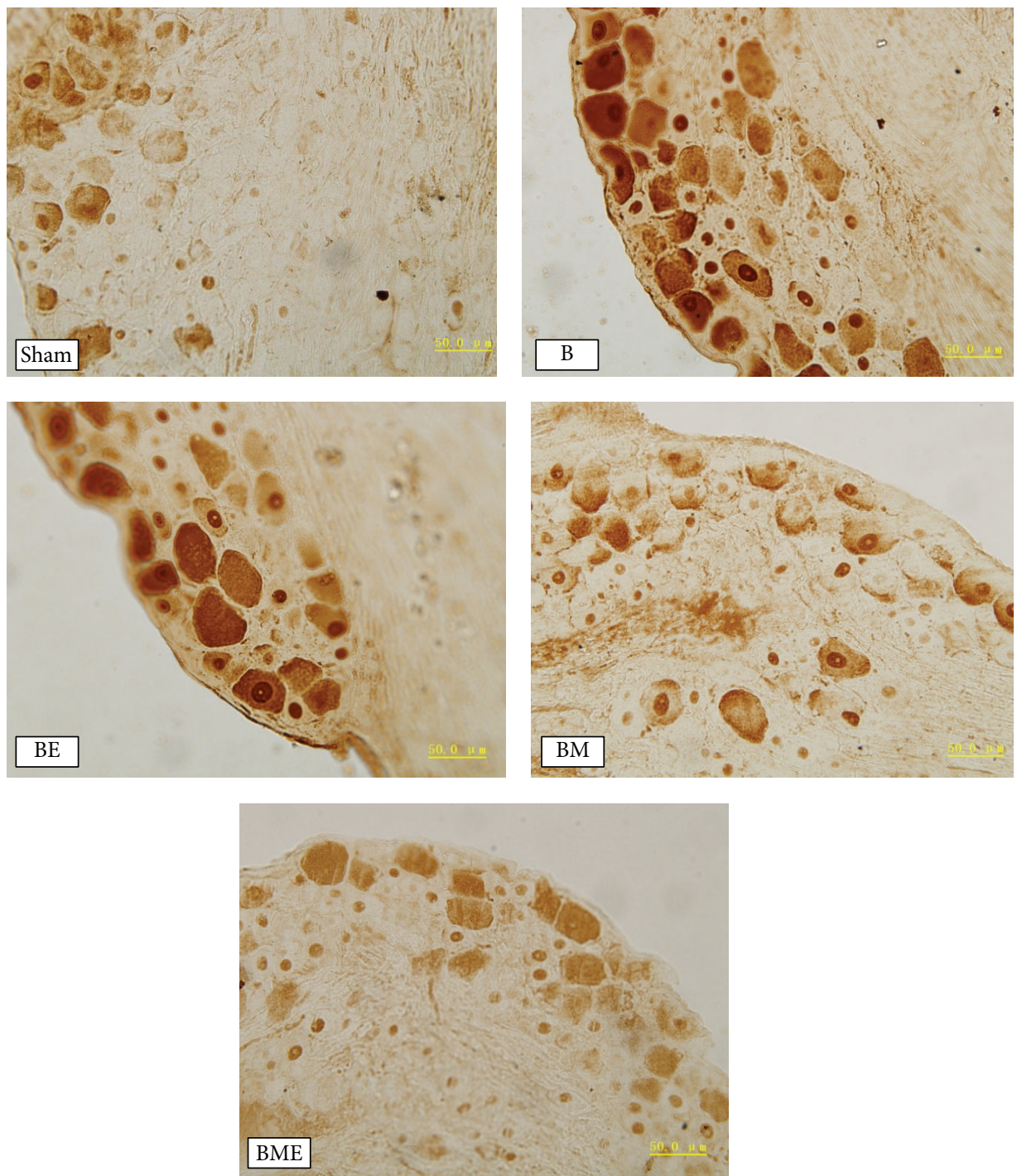

FIGURE 3: Expression of CGRP in dorsal root ganglion of the rats (CGRP staining, $\times 100$ ).

Previous studies have shown that electroacupuncture treatment could inhibit the thermal withdrawal response in the inflammatory pain-morphine tolerance models, and the underlying mechanisms are associated with the downregulation of the vanilloid receptor in the DRG and the inhibition of the expression of the CGRP and SP mRNA in the DRG [15]. CGRP mainly exists in the small or medium neurons in the DRG and is the most important neurotransmitter for the transmission of pain signals. However, using CGRP receptor antagonist CGRP8-37 could inhibit and reverse the morphine tolerance.

The findings of the present study showed that the effects of electroacupuncture treatment were similar to the application of CGRP8-37. We speculated that the mechanisms involved in the effects of electroacupuncture treatment on reducing the morphine tolerance were associated with the inhibition of the overrelease of CGRP in the DRG. Some other studies have also shown that electroacupuncture treatment could induce the release of endogenous opioid peptides, which participated in the phosphorylation and desensitization of $\mu$-receptor in neuropathic pain, thus inhibiting the occurrence of morphine tolerance [16].

This study found some evidences. First, electroacupuncture treatment alone for bone cancer pain could obtain analgesic effects only at early stage. The longer electroacupuncture and morphine treatment may lead to analgesic tolerance. Second, when used in combination with electroacupuncture, morphine treatment could result in improving the analgesic effects and reducing the tolerance. Third, the expression of CGRP in the DGR may associate with the pain behaviors.

Of course, we need more proof to support the correlative association between the electroacupuncture and the effects on CGRP. More studies are needed to further investigate inhibitor of CGRP in the electroacupuncture group to confirm the effect of CGRP for EA. Also, we need to explore whether electroacupuncture alleviated morphine tolerance has some relationship with other opioid receptor mechanisms [17]. 


\section{Competing Interests}

There are no financial disclosures for any of the authors.

\section{Acknowledgments}

This survey was supported by National Natural Science Foundation of China (no. 81403253).

\section{References}

[1] R. D. Rubens and R. E. Coleman, "Bone metastases", in Clinical Oncology, M. D. Abeloff, J. O. Armitage, A. S. Lichter et al., Eds., Churchill Livingstone, New York, NY, USA, 1995.

[2] P. Honore, S. D. Rogers, M. J. Schwei et al., "Murine models of inflammatory, neuropathic and cancer pain each generates a unique set of neurochemical changes in the spinal cord and sensory neurons," Neuroscience, vol. 98, no. 3, pp. 585-598, 2000.

[3] S. Mercadante and R. K. Portenoy, "Opioid poorly-responsive cancer pain. Part 1: clinical considerations," Journal of Pain and Symptom Management, vol. 21, no. 2, pp. 144-150, 2001.

[4] Z. Zuo, "The role of opioid receptor internalization and $\beta$ arrestins in the development of opioid tolerance," Anesthesia \& Analgesia, vol. 101, no. 3, pp. 728-734, 2005.

[5] T. Trang, R. Quirion, and K. Jhamandas, "The spinal basis of opioid tolerance and physical dependence: involvement of calcitonin gene-related peptide, substance $\mathrm{P}$, and arachidonic acidderived metabolites," Peptides, vol. 26, no. 8, pp. 1346-1355, 2005.

[6] C. A. Paley, M. I. Johnson, O. A. Tashani et al., "Acupuncture for cancer pain in adults," Cochrane Database of Systematic Reviews, vol. 10, article 7753, 2015.

[7] C. Hu, H. Zhang, W. Wu et al., "Acupuncture for pain management in cancer: a systematic review and meta-analysis," Evidence-Based Complementary and Alternative Medicine, vol. 2016, Article ID 1720239, 13 pages, 2016.

[8] C. A. Paley, M. I. Bennett, and M. I. Johnson, "Acupuncture for cancer-induced bone pain?" Evidence-Based Complementary and Alternative Medicine, vol. 2011, Article ID 671043, 8 pages, 2011.

[9] H. K. Ryu, Y.-H. Baek, Y.-C. Park, and B.-K. Seo, "Current studies of acupuncture in cancer-induced bone pain animal models," Evidence-Based Complementary and Alternative Medicine, vol. 2014, Article ID 191347, 8 pages, 2014.

[10] Q. F. Huang, "Exploration of clinical regularities in acupuncture-moxibustion treatment for cancer pain," Journal of Acupuncture and Tuina Science, vol. 9, no. 6, pp. 346-350, 2011.

[11] T. Chen, K. Wang, J. Xu, W. Ma, and J. Zhou, "Electroacupuncture reduces postoperative pain and analgesic consumption in patients undergoing thoracic surgery: a randomized study," Evidence-Based Complementary and Alternative Medicine, vol. 2016, Article ID 2126416, 7 pages, 2016.

[12] Y.-H. Gao, J.-Y. Wang, L.-N. Qiao et al., "NK cells mediate the cumulative analgesic effect of electroacupuncture in a rat model of neuropathic pain," BMC Complementary and Alternative Medicine, vol. 14, article 316, 2014.

[13] S. Zhang, H. Tang, J. Zhou, and Y. Gu, "Electroacupuncture attenuates neuropathic pain after brachial plexus injury," Neural Regeneration Research, vol. 9, no. 14, pp. 1365-1370, 2014.

[14] J.-W. Choi, S.-Y. Kang, J.-G. Choi et al., "Analgesic effect of electroacupuncture on paclitaxel-induced neuropathic pain via spinal opioidergic and adrenergic mechanisms in mice," The American Journal of Chinese Medicine, vol. 43, no. 1, pp. 57-70, 2015.

[15] L.-N. Qiao, J.-Y. Wang, Y.-S. Yang et al., "Effect of electroacupuncture intervention on expression of CGRP, SP, COX-1, and PGE2 of dorsal portion of the cervical spinal cord in rats with neck-incision pain," Evidence-Based Complementary and Alternative Medicine, vol. 2013, Article ID 294091, 9 pages, 2013.

[16] K. J. Powell, W. Ma, M. Sutak, H. Doods, R. Quirion, and K. Jhamandas, "Blockade and reversal of spinal morphine tolerance by peptide and non-peptide calcitonin gene-related peptide receptor antagonists," British Journal of Pharmacology, vol. 131, no. 5, pp. 875-884, 2000.

[17] M. Petraschka, S. Li, T. L. Gilbert et al., "The absence of endogenous $\beta$-endorphin selectively blocks phosphorylation and desensitization of mu opioid receptors following partial sciatic nerve ligation," Neuroscience, vol. 146, no. 4, pp. 17951807, 2007. 


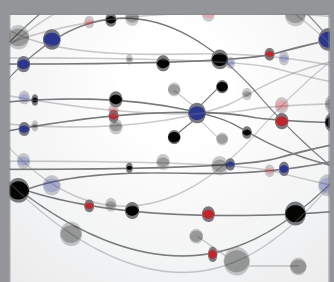

The Scientific World Journal
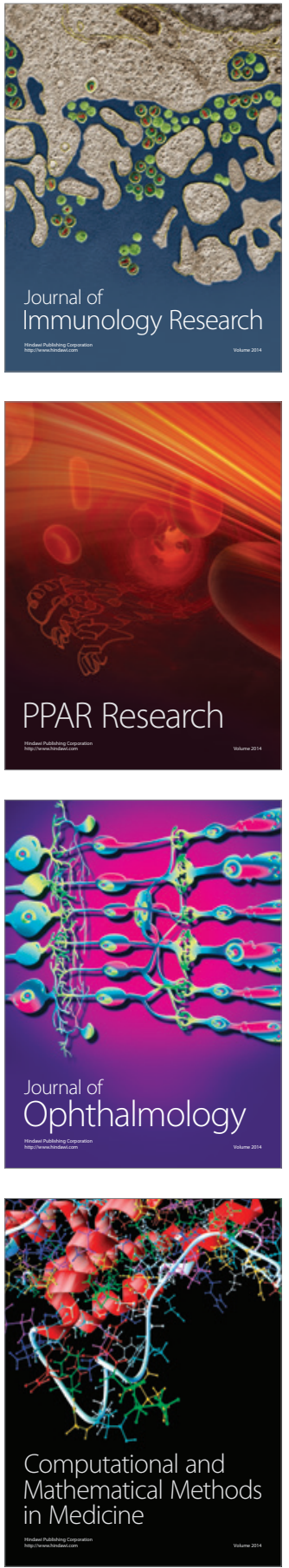

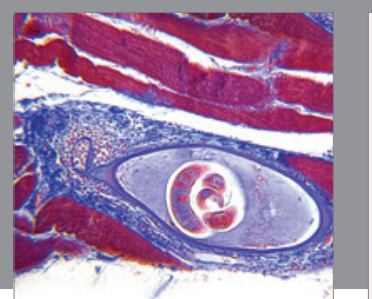

Gastroenterology Research and Practice

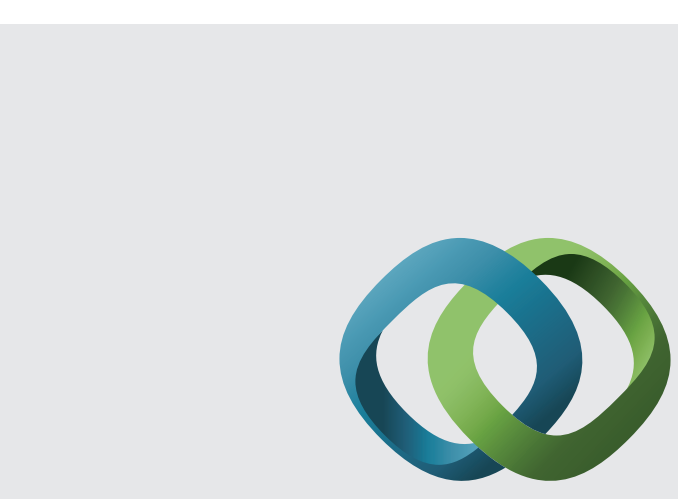

\section{Hindawi}

Submit your manuscripts at

http://www.hindawi.com
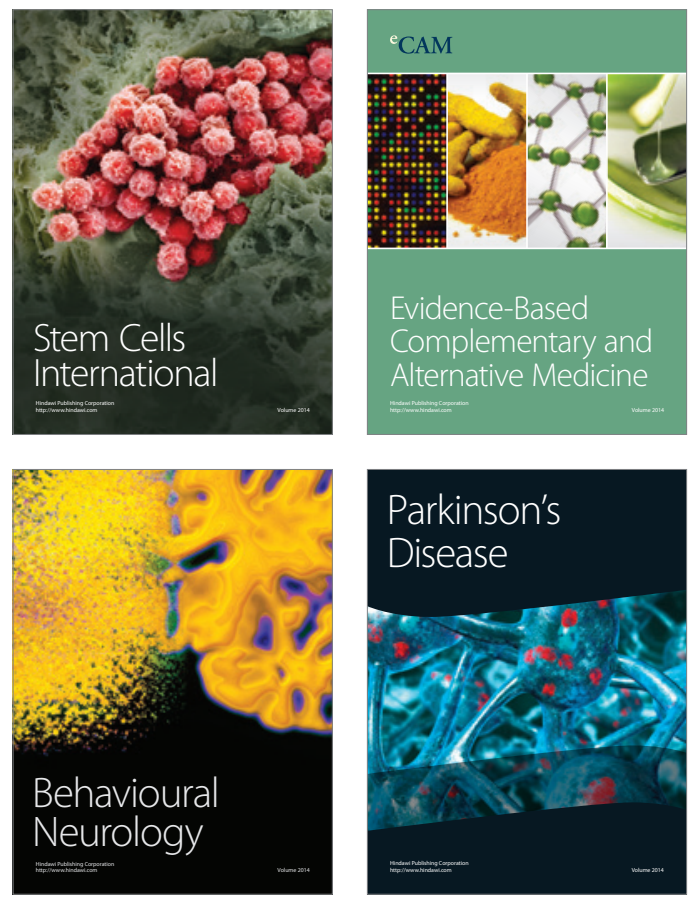
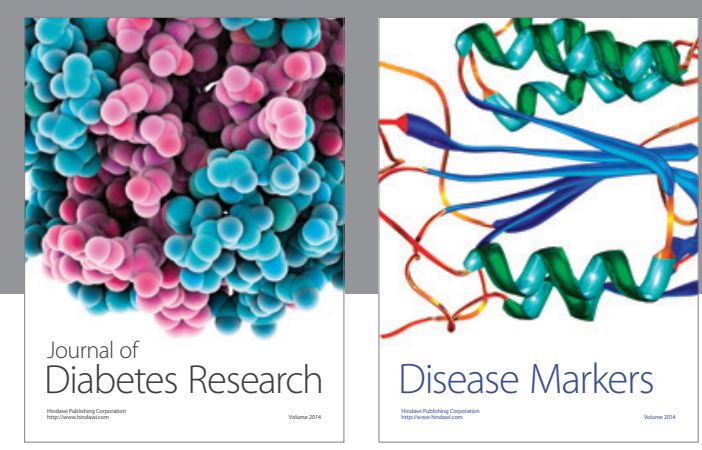

Disease Markers
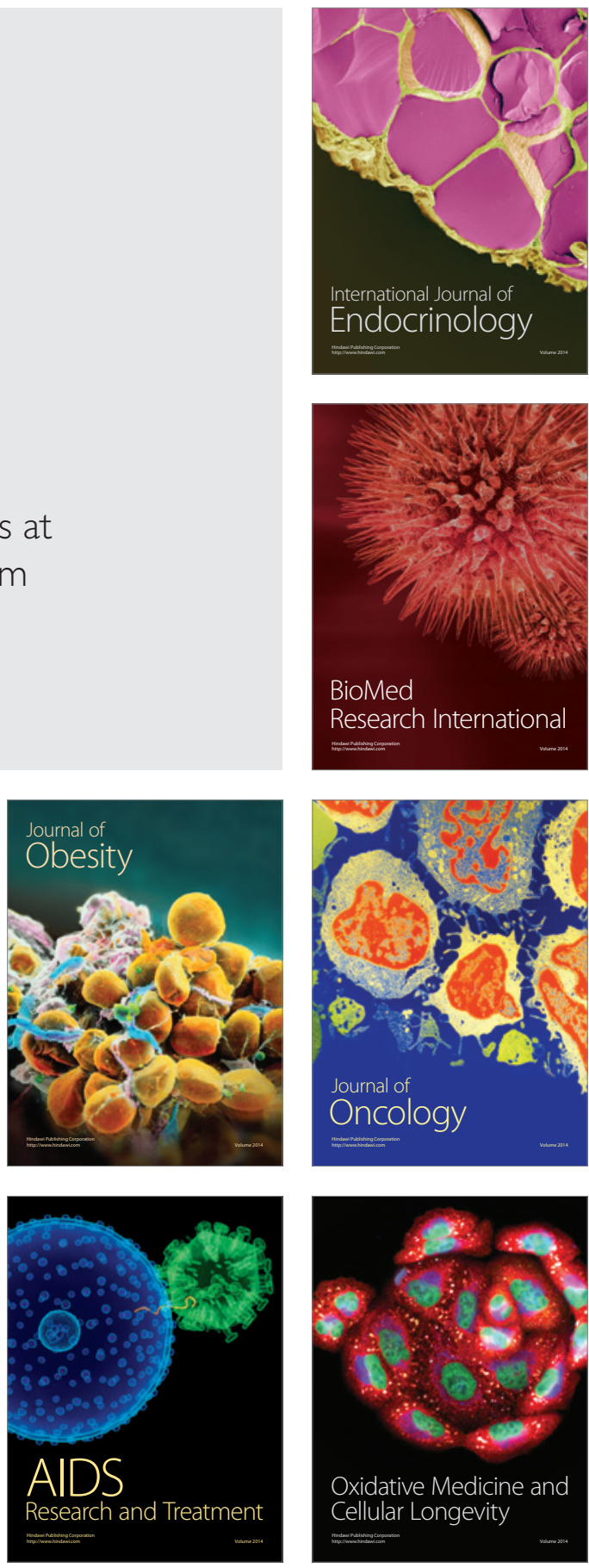